Submission ID: 43901

\title{
The Qualitative Accuracy Estimation of Velocity Model in Case of a Complex
} Geology

E.L. Svistova* (PetroTrace), A.E. Korolev (PetroTrace), F.F. Zeleznyak (PetroTrace), E.M. Rossiyskaya (PetroTrace), A.A. Shevchenko (PetroTrace)

\section{SUMMARY}

The main idea of the seismic data processing is to obtain a true geological model and final reserves estimate. Apart from the geological and geophysical parameters, structural characteristics, such as the size and position of structures in space, undoubtedly influence the amount of reserves. In areas with complex geological structure, the time and depth scale do not correspond to each other. Uncertainties in geological characteristics can lead to large errors in the estimation of reserves. Thus, reliable depth imaging of the environment(media) is one of the main tasks, while the uncertainty of structural constructions can be quite large, depending on the complexity of the geological structure.

The work demonstrates the interpretational approach to the process of the velocity model building and the method of qualitative accuracy estimation of velocity model in case of complex geological conditions. 


\section{Качественная оценка точности построения глубинной скоростной модели среды в сложных геологических условиях}

Свистова Е.Л.* (ООО «ПетроТрейс»), Зелезняк Ф.Ф. (ООО «ПетроТрейс»), Королев А.Е. (ООО «ПетроТрейс»), Российская Е.М., Шевченко А.А. (ООО «ПетроТрейс»)

Основной задачей сейсморазведки является получение геологической модели и подсчет запасов. На величину запасов наряду с геолого-геофизическими параметрами среды, несомненно, влияют и структурные характеристики, такие как размер и положение структур в пространстве. Неопределенности во всех этих геологических характеристиках могут привести к большим погрешностям в оценке запасов. Таким образом, получение достоверного глубинного изображения среды является одной из главных задач, при этом неопределенность структурных построений может быть достаточно большой в зависимости от сложности геологического строения среды.

\section{Введение}

Несмотря на то, что большая часть сейсмической интерпретации делается по разрезам во временном масштабе, структурную интерпретацию надежнее выполнять в глубиной области. Скоростные неоднородности в среде могут создавать на временном разрезе ложные структуры, которые зачастую очень сложно отделить от настоящей геологии. Преобразование в глубинную область с корректной скоростной моделью позволяет уменьшить неопределенность в структурном плане, свойственную временному представлению.

Различные методы преобразования время-глубина $[1,3]$ могут быть разделены на две категории: прямое или одномерное преобразование время- глубина (перемещение отражений по вертикали) и миграционное преобразование, основанное на одновременном изменении вертикального масштаба и горизонтального смещения (миграции) отражений. При миграционном преобразовании оптимальны скорости подбираются исходя из хорошей фокусировки изображений и корректного положения отражающих границ после миграции. Скорости миграции подбираются тестированием моделей. Критерием качества выступает качество фокусировки отражений и только потом правильное положение границ в сопоставлении со скважинной информацией.

Выполнение глубинной миграции до суммирования для получения хорошего сейсмического разреза один из наиболее трудоемких шагов обработки сейсмических данных. Тщательно построенные глубинно-скоростные модели (ГСМ) и современные методы миграционных преобразований позволяют получить изображения, характеризующиеся хорошей фокусировкой отражающих границ и в существенной степени приближенные к реальной глубинной геометрии. Для получения глубинно-скоростных моделей имеются большие возможности, в том числе позволяющие получить хорошую корреляцию со скважинами данными.

Особая необходимость в миграционных преобразованиях возникает тогда, когда имеются отличные от нуля наклоны границ и когда скорости в среде характеризуются существенными изменениями по латерали. Трансформация сейсмического изображения при миграции происходит по криволинейному лучу с учетом преломления на отражающих границах. Из-за преломления лучей сигнал, посланный вертикально, вряд ли будет распространяться и далее вертикально, т.е. скорости, обеспечивающие наилучшее спрямление годографа отраженных волн, оптимальную фокусировку, и миграцию, не совпадают с вертикальными скоростями, обеспечивающими истинные глубины отражающих горизонтов. 
Al-Chalabi в своей статье [2] следующим образом охарактеризовал 'скорость', полученную в результате сейсмической обработки: “имеет размерность скорости, но в общем или только отдаленно или весьма неопределенно имеет отношение к фактической скорости распространения волн в земле. Наиболее распространенный тип такой 'скорости' - то, что обычно известно, как скорость оптимального суммирования. Eе действительное предназначение - то, что она есть параметр, обеспечивающий оптимальное выравнивание однократных отражений на подборках СТО, и только это. Точно также, 'скорости', полученные путем применения различных способов анализа скоростей миграции до суммирования, есть, прежде всего, параметры, которые обеспечивают оптимальную фокусировку мигрируемой энергии. Вообще говоря, все эти параметры никак не представляют скорости в земле".

Получается, что скорости миграции не годятся для истинного преобразования времяглубина и наоборот, вертикальные скорости преобразующие времена в истинные глубины не пригодны для миграционных преобразований.

\section{Методы сопоставления глубинных и временных изображений}

Результат глубинной миграции получается в области глубин, полученное изображение содержит невязки со скважинными отбивками глубин отражающих горизонтов. Причины невязок могут быть различными. Восстановление модели среды по наблюденным данным - это классическая обратная задача. Теоретически существует много скоростных моделей, которые будут производить эквивалентное изображение [4]. С другой стороны, ошибки измерения, которые никогда неизвестны, но должны быть учтены при структурных построениях. При выполнении практических проектов необходимо решение, которое бы давало возможность на этапе интерпретации получить скоростную модель среды, отражающую плавные или «загрубленные» изменения скоростей, наблюдаемые на скоростях миграции и, с другой стороны, давало бы некоторое корректное сопоставление временных и глубинных масштабов.

Временная миграция строго применима только для вертикально изменяющейся скорости; она не учитывает преломления луча на поверхностях раздела. Глубинная миграция учитывает преломление луча на границах раздела, но требует точную скоростную модель. Глубинная скоростная модель для PSDM обычно отличается от RMS скоростей, используемых для PSTM, большей детальностью. Такая ГСМ позволяет учесть влияние локальных приповерхностных неоднородностей, сложной конфигурации отражающих горизонтов, а также, сильноконтрастных скоростных неоднородностей на нижележащие горизонты. По мнению многих авторов, хорошим подходом к преобразованию в глубину, особенно в сложных геологических условиях, является следующая последовательность действий $[1,3]$ :

1. выполнение глубинной миграции со скоростной моделью, оптимальной для получения качественного изображения;

2. трансформация полученного мигрированного изображения во времена и обратное преобразование время-глубина с использованием «загрубленных» скоростей миграции, полученных с помощью интерпретационных действий (осреднения, коррекции);

Существует много подходов конверсии в глубину и каждый имеет свои собственные преимущества и недостатки. Однако «истинные» вертикальные скорости имеют высокую степень надежности только в известных точках расположения скважин. Дополнительная информация о поведении скоростей в межскважинном пространстве может быть взята из сейсмических скоростей. В работе демонстрируется интерпретационный подход к построению 
скоростной модели и метод качественной оценки точности построения глубинной скоростной модели среды в сложных геологических условиях.

В ходе работ по нескольким производственным проектам выполнялось моделирование и практические действия проверялись на модельных данных. Основные этапы работы демонстрируются на разрезе, в котором существует высокоскоростной пласт в ВЧР и имеются небольшие по размеру скоростные аномалии на средних глубинах. На Рис.1А. показана скоростная модель, подобранная для целей качественного построения изображения при глубинной миграции. В ГСМ видны резкие латеральные изменения скоростей, размер которых достаточно мал по сравнению с сейсмически обоснованной горизонтальной разрешающей способностью. Появление таких скоростных аномалий обусловлено повышением качества фокусировки изображения. При использовании такой скоростной модели при преобразовании время-глубина и сопоставление результатов временных и глубинных моделей становится некорректным. Интерпретационный подход к сглаживанию скоростной модели среды приводит к получению модели $\mathrm{c}$ плавными латеральными вариациями скоростей. Сопоставление двух скоростных моделей дается на Рисунке 2. На Рис.2В показан разрез во временном масштабе, полученный из глубинного масштаба мигрированного (PSDM) изображения. При преобразовании использовалась сглаженная скоростная модель (Рис.2Б). При этом поведение основных отражающих горизонтов не имеет резких флуктуаций, которые возникают при использовании скоростей миграции (Рис.1А).

Использование термина «интерпретация скоростной модели среды» может вызвать дискуссию о том, что существует возможность внести дополнительные субъективные ошибки в преобразование данных. Ошибки преобразования глубина- время могут привести к изменению положения отражающих горизонтов во временно масштабе. Поэтому структурные построения должны делаться по исходному глубинному изображению. Временное представление изображений может использоваться для анализа динамики волнового поля и интерпретации изменения различных атрибутов, рассчитываемых по разрезам и кубам во временном масштабе.
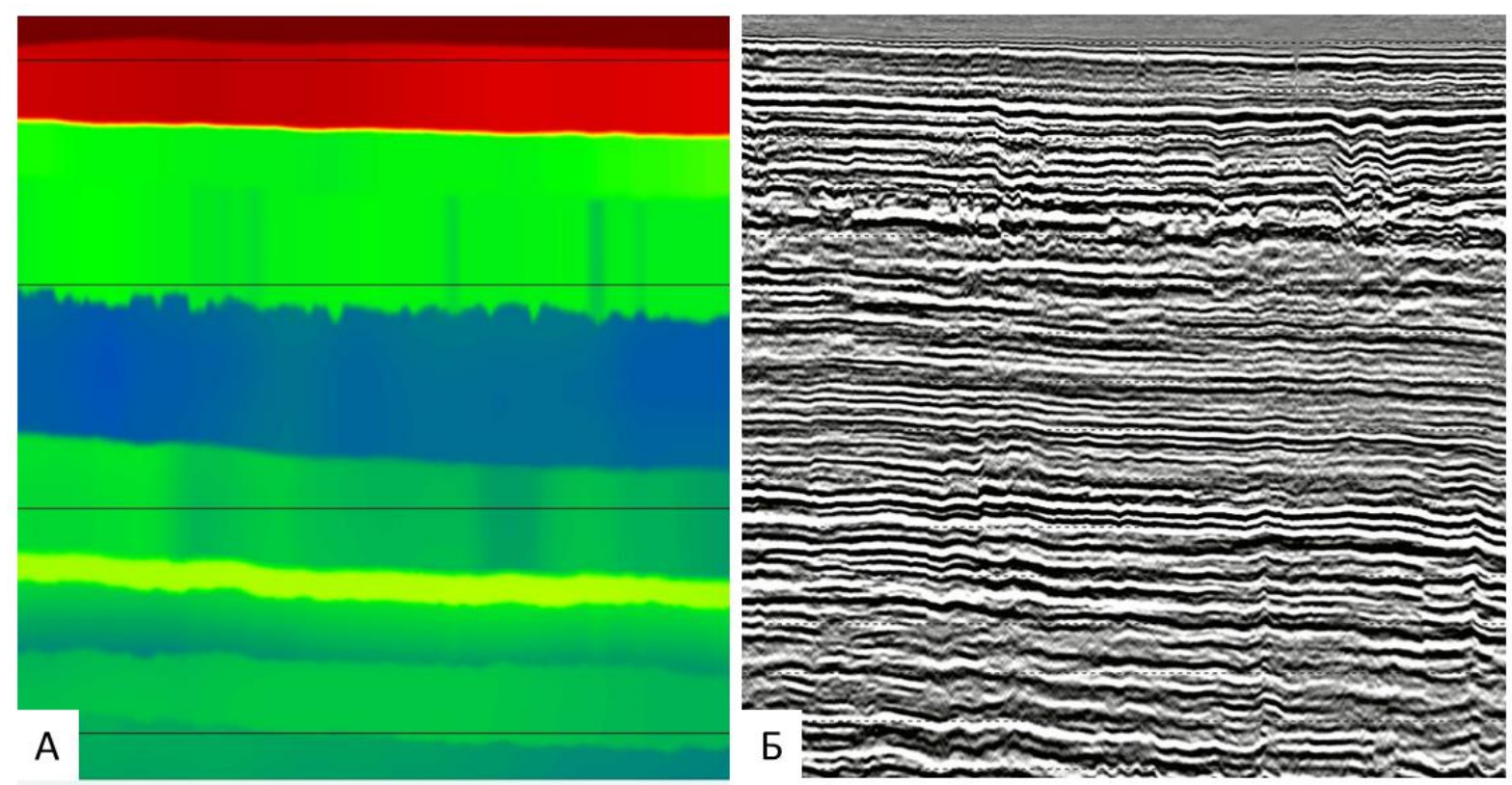

Рисунок 1 Скоростная модель (А) и изображение после глубинной миграџии (Б) 


\section{EAGE}
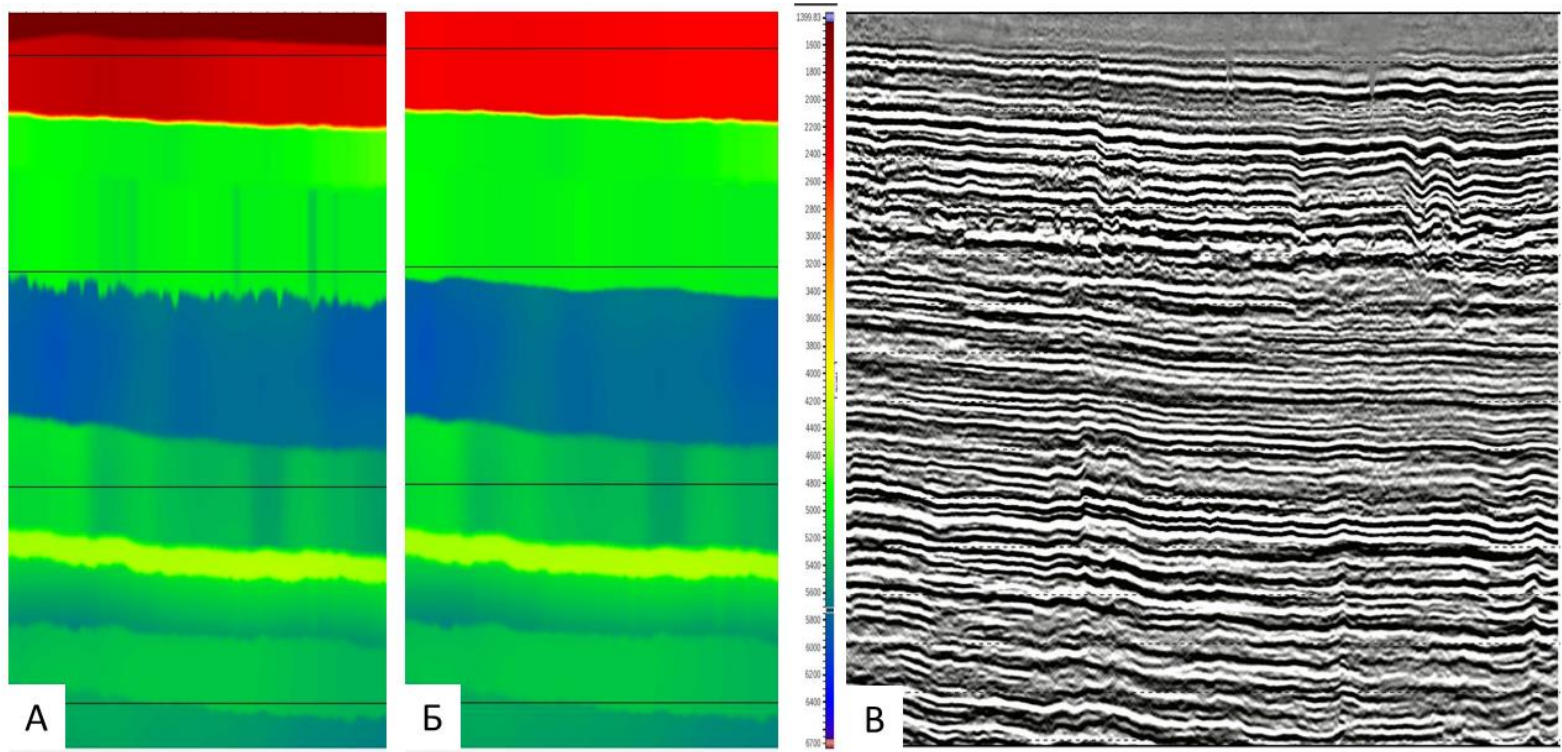

A

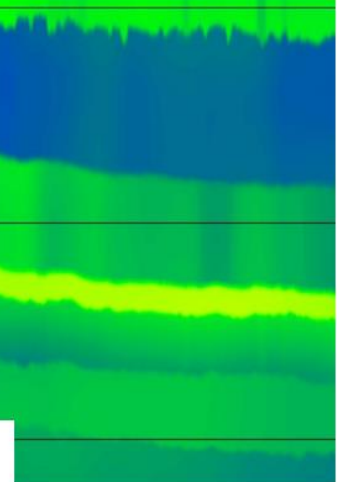

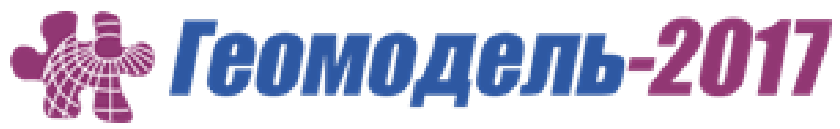

Рисунок 2 Скоростные модели и мигрированное (PSDM) изображение во временном масштабе. А - скоростная модель глубинной миграции (PSDM); Б- Временная модель после интерпретации (замещение ВЧР и сглаживание контрастного горизонта); $B$ Мигрированное изображение (PSDM) пересчитанное во временной масштаб.

\section{Выводы}

Исследования на моделях и работа в рамках производственных проектов показывает, что скоростные модели, построенные для выполнения глубинных миграционных преобразований, имеют «завышенную» латеральную и вертикальную разрешенность. Использование таких моделей дает хорошее миграционное изображение, но использование данной модели при сопоставлении временных и глубинных изображений неприменимо. Для преобразования изображений из глубинного масштаба во временной требуется более «гладкая» скоростная модель. Для построения такой модели среды необходимо выполнить интерпретацию модели, включающую в себя учет скоростных аномалий в ВЧР и загрубление модели с использованием информации о модели эффективных скоростей (построенной для миграции данных PSTM). Построенная «загрубленная» скоростная модель дает возможность сопоставления глубинных и временных масштабов без применения процедуры сноса.

\section{References:}

1. True depth conversion: More than a pretty picture

Edward L. Etris, Nick J. Crabtree, Jan Dewar, Scott Pickford, A Core Laboratories Company

2. Seismic velocities - a critique

Al-Chalabi, M., 1994, , First Break, 12, no. 12, 589-596

3. Depth prediction from a prestack depth image: a Dutch North Sea case study

Crabtree, N., Hill, D., Veltmeijer, H., 2001, SEG Annual Meeting, San Antonio, September 2001

4. Investigating the velocity-depth ambiguity of reflection traveltimes

Tieman, H.J., 1994, Geophysics, 59, no. 11, 1763-1773 